\title{
Anatomia de um Caso de Dextrocardia com Situs Solitus
}

\author{
Anatomy of a Dextrocardia Case with Situs Solitus
}

Fabíola Sawaguchi Faig-Leite ${ }^{1}$ e Horácio Faig-Leite ${ }^{2}$

Universidade Estadual Paulista Júlio de Mesquita Filho (UNESP) Campus de Botucatu', Botucatu, SP; Universidade Estadual Paulista Júlio de Mesquita Filho (UNESP) Campus de São José dos Campos², São José dos Campos, SP - Brasil

Apresentamos um caso raro de dextrocardia em um cadáver feminino com idade aproximada de um ano, apresentando situs solitus. Normalmente, os casos de dextrocardia relatados na literatura descrevem múltiplas malformações cardíacas associadas. No presente estudo, tem grande relevância o fato de não ter sido encontrada uma completa imagem especular do coração e dos vasos da base. Não foram encontradas discordância atrioventricular ou outras malformações intra e extracardíacas que são comumente incidentes em casos de dextrocardia. No entanto, foram encontradas a presença de um grande ducto arterioso, bem como uma posição anômala da parte direita do coração.
We present a rare case of dextrocardia in the corpse of a female child, aged approximately one year old, presenting situs solitus. The cases of dextrocardia reported in the literature normally describe multiple associated cardiac malformations. In the present study, it is noteworthy the fact that a complete specular image of the heart and the vessels of the base of the heart was not found. There was no atrioventricular discordance or other intra and extracardiac malformations, which are commonly seen in cases of dextrocardia. A large ductus arteriosus was found, as well as the anomalous position of the right portion of the heart.

\section{Introdução}

A dextrocardia é uma malformação embriológica que se caracteriza pelo deslocamento do eixo maior (base ao ápice) do coração para o lado direito do tórax com reversão da inclinação apical. Essa malformação decorre de uma rotação anômala do tubo primitivo do coração para a esquerda, na qual o laço bulboventricular se dobra à esquerda, em uma imagem especular do normal o que ocorre por volta da oitava semana de vida embrionária ${ }^{1,2}$.

A incidência da dextrocardia associada a situs inversus na população em geral é de 1:10.000, enquanto a associada a situs solitus é de 1:30.000 em nascidos vivos e de somente 1:900.000 na população adulta ${ }^{3,4}$. Essa grande diferença de incidência encontrada em indivíduos com dextrocardia associada a situs solitus se deve a maior ocorrência de doenças cardíacas e/ou extracardíacas associadas, como fístula traqueoesofágica, hipoplasia pulmonar, ânus imperfurado, espinha bífida e síndrome de Kartagener ${ }^{3-6}$.

Existe uma grande divergência entre os autores no que diz respeito à classificação dos diversos tipos de dextrocardia ${ }^{1,6-8}$.

Dentre os vários trabalhos encontrados, destaca-se o de Van Praagh e cols. ${ }^{6}$ por descrever e classificar de forma mais detalhada os diferentes tipos de dextrocardia. A dextrocardia deve ser diferenciada da dextroversão, uma vez que esta é uma condição não congênita, na qual o coração pode mudar

\section{Palavras-chave}

dextrocardia, dissecção, ducto torácico, defeitos dos septos cardíacos.

\section{Correspondência: Fabíola Sawaguchi Faig Leite•}

Rua Engenheiro João Fonseca dos Santos, 123 / 194, Vila Adyanna, 12.243-

620, São José dos Campos, SP - Brasil.

E-mail: fasawaguchi@yahoo.com.br

Artigo recebido em 11/01/08; revisado recebido em 24/02/08; aceito em

10/03/08.; de lado, em decorrência de uma doença que empurre ou puxe este órgão para o antímero direito, todavia o ápice cardíaco continua voltado para o lado esquerdo $2,5,7,9,10$.

\section{Relato do Caso}

Descrição anatômica

A dextrocardia foi encontrada em um cadáver de uma criança do sexo feminino, com idade provável de um ano, pertencente ao Laboratório de Anatomia do Campus de São José dos Campos - UNESP.

Na peça em questão, a região do tórax encontrava-se parcialmente dissecada, sem a presença do pericárdio e dos pulmões. O osso esterno encontrava-se em sua posição normal. A inspeção do coração e dos vasos da base não revelou possíveis alterações de posicionamento decorrente da retirada dos pulmões. Todo o coração e os vasos da base estavam íntegros e não haviam sido dissecados. O coração e o seu ápice estavam totalmente voltados para a direita.

Removido o esterno e dissecados os vasos da base, observou-se que, devido à rotação que o coração apresentava, toda a porção direita do coração estava deslocada mais para a parte posterior, sendo que tal fato era tão acentuado na aurícula direita, que estando o coração na posição encontrada, não se conseguia visualizá-la (fig. 1). Essa rotação anormal do coração fez com que o tronco pulmonar emergisse do ventrículo direito de uma forma anômala, apresentando-se muito mais superficial e extenso (fig. 1).

Durante a inspeção do tronco pulmonar foi encontrado um grande ducto arterial (ducto de Botallo) (fig. 2). O ápice cardíaco encontrava-se posicionado ao longo do eixo do coração e voltado para o lado direito evidenciando, assim, a dextrocardia.

Com o auxílio de uma lupa esterioscópica, todos os ramos aferentes e eferentes dos vasos da base foram dissecados para se verificarem anomalias. Com exceção do tronco 


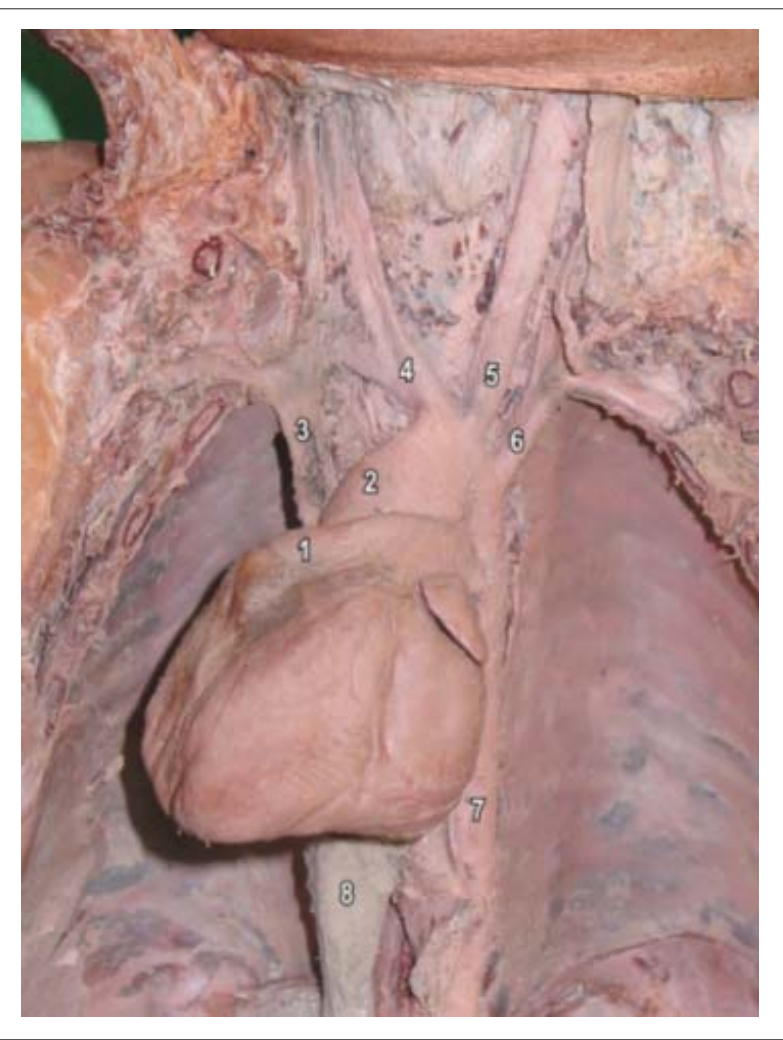

Fig. 1 - Coração em posição no tórax : 1 - tronco pulmonar; 2 - aorta ascendente; 3 - veia cava superior; 4 - tronco braquiocefálico; 5 - artéria carótida comum esquerda; 6 - artéria subclávia esquerda; 7 - aorta torácica; 8 - tecido hepático recobrindo a veia cava inferior.

braquiocefálico, que apresentava uma angulação mais acentuada que o normal, nenhuma outra variação de posição ou trajeto foi encontrada (fig. 1).

Foi removido o músculo diafragma e, em seguida, dissecado o fígado, que apresentava aspecto e morfologia normal. A cavidade abdominal foi aberta, e todas as vísceras se encontravam normalmente localizadas, não apresentando nenhum tipo de alteração, o que caracterizava situs solitus.

Terminada essa fase, o coração foi, então, cuidadosamente removido do seu local e novamente inspecionado pelo uso da lupa. As aurículas e os átrios foram abertos e analisados e revelaram aspectos anatômicos normais. A concordância átrioventricular era anatomicamente normal. Foi feita uma incisão em cada ventrículo, com o objetivo de verificar sua morfologia interna e a emergência dos seus vasos. Os dois ventrículos apresentavam a morfologia interna, as valvas átrio-ventriculares e a emergências dos vasos normais. A aorta e o tronco pulmonar apresentavam as valvas semilunares normais.

\section{Discussão}

A literatura apresenta uma grande diversidade de tipos e classificações para os casos de dextrocardia. Essa diversidade parece decorrer do fato de ser difícil estabelecer critérios para uma perfeita classificação ${ }^{6-8}$.

Pela pura observação anatômica pode-se constatar que

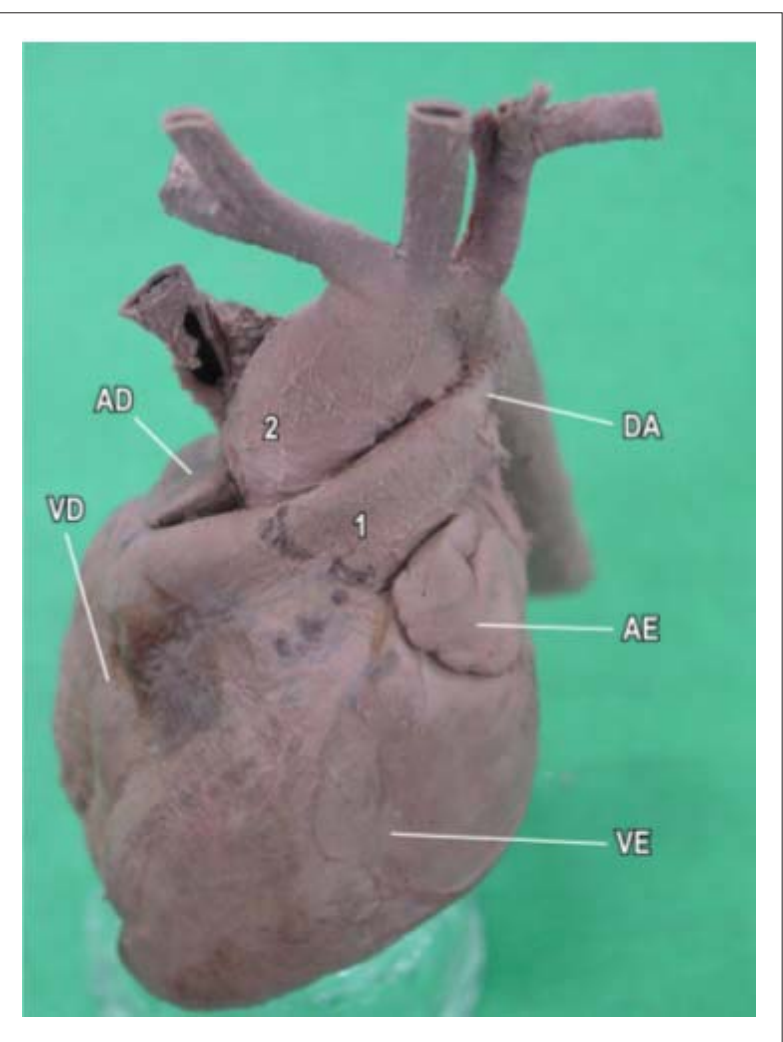

Fig. 2 - Coração isolado mostrando as câmaras cardíacas: $A D$ - aurícula direita; $V D$ - ventrículo direito; $A E$ - aurícula esquerda; VE - ventrículo esquerdo; $D A$ - ducto arterial; 1 - tronco pulmonar; 2 - aorta ascendente.

não houve mudança de localização do coração após a fixação do cadáver.

Este caso caracteriza-se como dextrocardia uma vez que o ápice cardíaco estava completamente voltado para o antímero direito ${ }^{2-5,7,9}$. Não pode ser classificado como uma dextroversão pois nesta, o coração localiza-se no hemitórax direito com o seu ápice voltado para o antímero esquerdo, diferente do que se observa no presente $\mathrm{Caso}^{5,9}$ (fig. 1).

O trabalho de Van Praagh e cols. ${ }^{6}$ é o único que descreve dois casos idênticos ao caso, em relato com maior riqueza de detalhes anatômicos. Ambos, como neste caso, constituíam necropsias de crianças menores de 1 ano de idade e portadoras de um tipo de dextrocardia classificada pelos autores como dextrocardia com grandes vasos normalmente relacionados. Nesses casos, assim como no caso em apresentação, foi encontrado situs solitus, os grandes vasos normalmente relacionados (com o laço bulboventricular para a direita) e a ausência de inversão ventricular. Os dois casos descritos por esses autores apresentavam graves alterações pulmonares; no caso relatado, como os pulmões já haviam sido retirados, não nos foi possível estudá-los.

Calcaterra e cols. ${ }^{7}$, estudando vários casos de dextrocardia, descreveram um caso com as mesmas variações anatômicas por nós encontradas; os autores, contudo, não pesquisaram alterações extracardíacas nas necropsias, não permitindo, assim, uma comparação mais detalhada com o presente caso. 


\section{Relato de Caso}

Normalmente, a dextrocardia é mais freqüente quando associada a situs inversus, apresentando ou não malformações cardíacas e/ou extracardíacas associadas ${ }^{4,9}$. O caso apresentado é mais incomum por estar associado a situs solitus e por não apresentar esses tipos de malformações, o que nos permite afirmar que esta variedade de posição cardíaca não está necessariamente associada a anomalias nas comunicações átrio-ventriculares, nas relações entre o coração e seus vasos aferentes e eferentes ou mesmo na função desse órgão.

O diagnóstico de dextrocardia pode ser feito durante o exame físico, por meio de um eletrocardiograma ou de um exame por imagem ${ }^{4}$. Portanto, o conhecimento dessa anomalia e de suas variáveis é de fundamental importância aos médicos em virtude do risco de apresentações atípicas de angina, de sua associação freqüente com outras doenças cardíacas e/ou extracardíacas e, também, por constituir uma malformação cujas anormalidades, a despeito de sua complexidade, atualmente são passíveis de correção cirúrgica ${ }^{8,9}$.

\section{Potencial Conflito de Interesses}

Declaro não haver conflito de interesses pertinentes.

\section{Fontes de Financiamento}

O presente estudo não teve fontes de financiamento externas.

\section{Vinculação Acadêmica}

Não há vinculação deste estudo a programas de pósgraduação.

\section{Referências}

1. Macruz R, Mazzieri R, Mattar Jr J, Ebaid M. Más posições cardíacas. Arq Bras Cardiol. 1973; 26: 481-6.

2. Pego-Fernandes PM, Serro-Azul, JB, Matheus F, Maehara BS. Revascularização do miocárdio em paciente com situs inversus totalis. Arq Bras Cardiol. 2007, 88 (5): e103-e106.

3. McCasckie AW, Thompson MM, Underwood MJ, Pallot DJ. A case of dextrocardia with normal situs. Acta Anat. 1991; 142: 288-92.

4. Bohun CM, Potts JE, Casey BM, Sandor GGS. A population-based study of cardiac malformations and outcomes associated with dextrocardia. Am J Cardiol. 2007; 100: 305-9.

5. Leung AKC, Robson WLM. Dextrocardia with sinus solitus. Can Med Assoc J. $2006 ; 175$ (3): 244.
6. Van Praagh R, Van Praagh S, Vlad P, Keith JD. Anatomic types of congenital dextrocardia; diagnostic and embryologic implications. Am J Cardiol. 1964; 13: 510-31.

7. Calcaterra G, Anderson RH, Lau KC, Shinebourne EA. Dextrocardia-value of segmental analysis in its categorization. Br Heart J. 1979; 42: 497-507.

8. Stanger P, Rudolph AM, EdwardsJE. Cardiac malpositions; an overview based on study of sixty-five necropsy specimens. Circulation. 1977; 56 (2): 159-72.

9. Garg N, Agarwal BL, Modi N, Radhakrishnan S, Sinha N. Dextrocardia: an analysis of cardiac structures in 125 patients. Int J Cardol. 2003; 88: 143-55.

10. Lucchese FA, Becker AE, Macartney FJ, Meier MA, Jimenez MQ, Shinebourne EA, et al. Classificação das cardiopatias congênitas. Arq Bras Cardiol. 1980; 35 (5): 427-34. 